\title{
SEROLOGIC LOCALIZATION OF ORGANIC BRAIN LESIONS *
}

\author{
J. M. RETINGER. Ph.D \\ CHICAGO
}

During my work on dementia precox, the dialysis method of Abderhalden, slightly modified, proved to be of great value. Yet, as this method did not, for certain reasons, become very popular, I thought it imperative to show that if used properly it is quite reliable and more than that, it can be of great value in the clinical diagnosis of obscure cases.

It seemed advisable to attempt to localize by this method those gross brain lesions which could be verified either by an operation or by necropsy, or by unmistakable clinical evidence. The technic used in all experiments was a modification of the original method based on nearly 4,000 tests, was easy to perform, less complicated and perfectly reliable. The detailed description of this modification will be found in the latter part of this article.

In the cases in which the dialysis method has been used for the purpose of a focal brain diagnosis, twenty-five have been sufficiently studied clinically or verified by operation or necropsy to be considered. The clinical diagnosis or symptoms were not known to me in most of the cases of this series. The serum was delivered for examination with only the general information that the patient suffered from some brain lesion, the location of which was not even suggested, but which I was requested to locate by serologic means. After the test was made and the diagnosis from the test was noted, it was given to the clinician for final comparison.

In the general table (Table 1) summarizing the results, the portions of the brain ("substrates") taken for the single tests are named by Roman and Arabic numbers. Figures 1 and $2^{1}$ show the gyri which correspond to the numbers in Table 1 and in the text. Table 2 represents the subcortical structures under III and IV.

* Submitted for publication March 12, 1918.

* From the Psychopathic Hospital of Cook County.

* Part of the work reported in this paper was conducted in the Otho S. A. Sprague Memorial Institute; another part in the Memorial Institute for Infectious Diseases.

1. Figures 1 and 2 are adapted from pictures in the "Human Anatomy" of Spalteholz. 


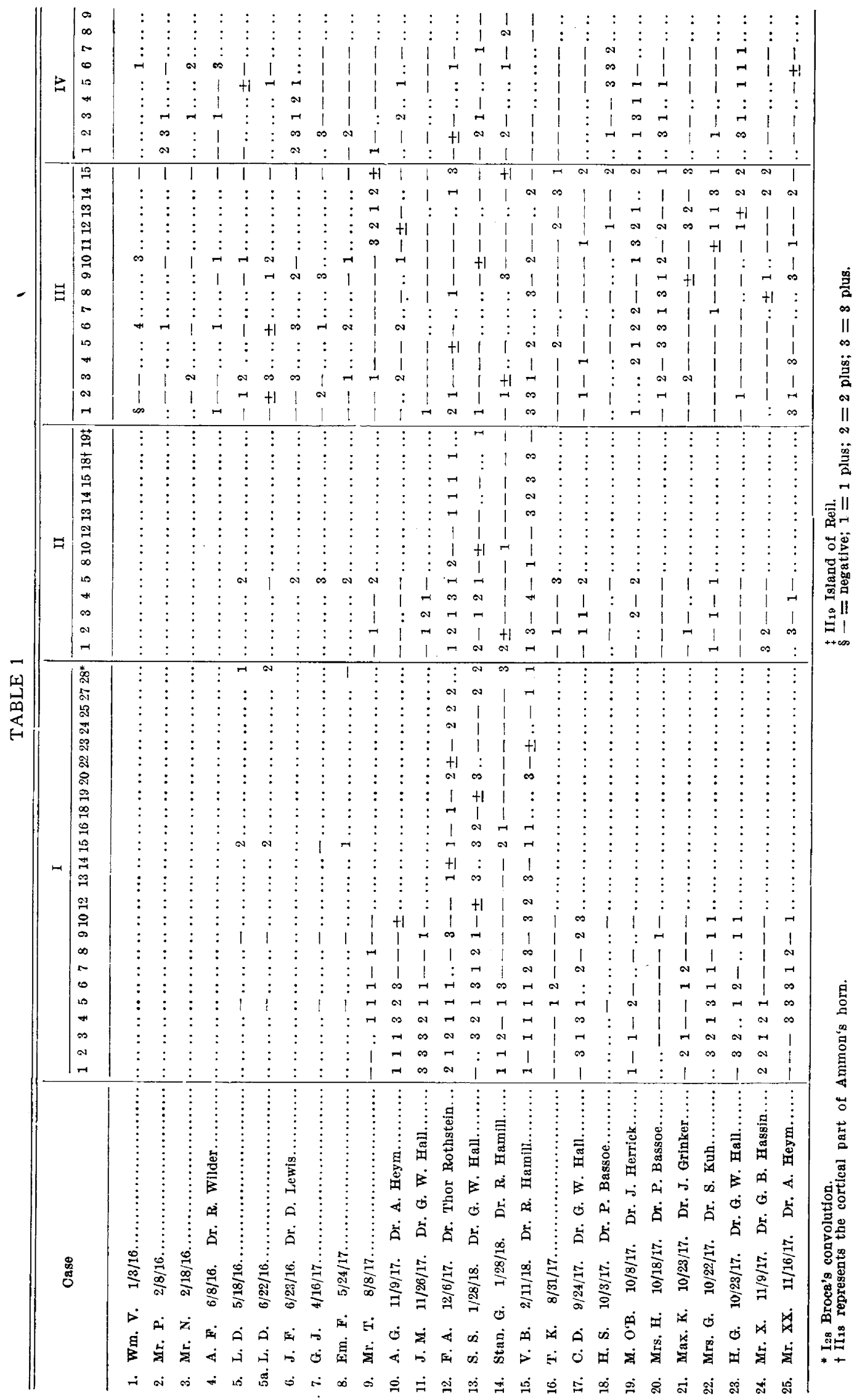




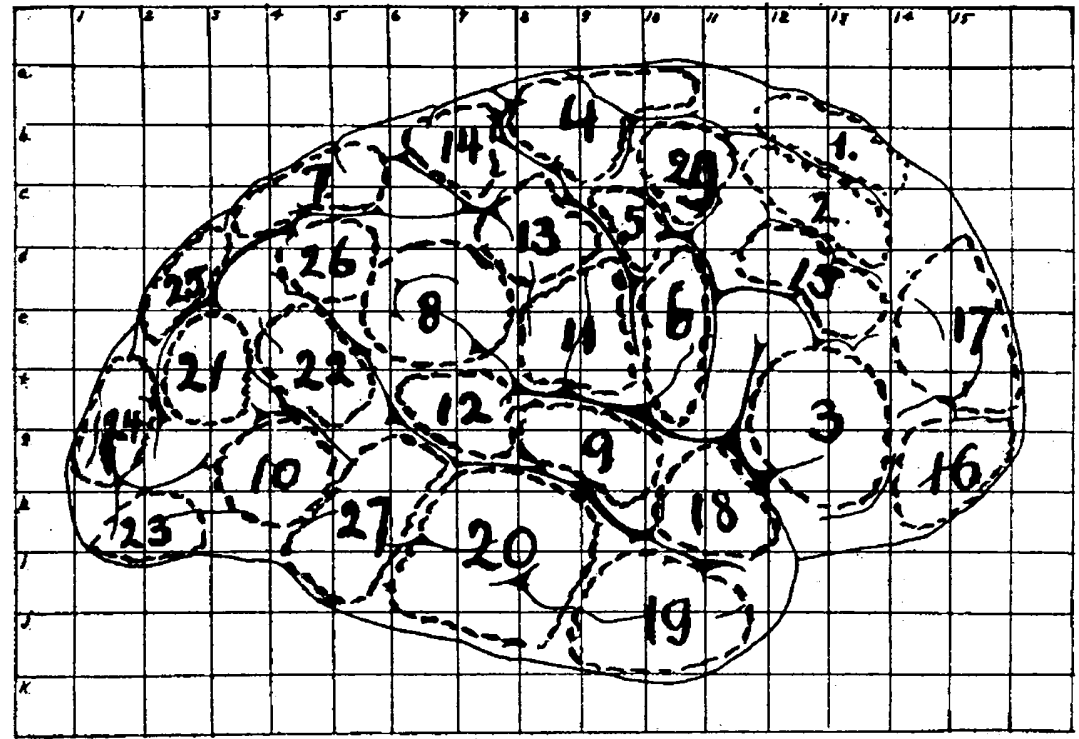

Fig. 1.-The areas of the brain cortex.

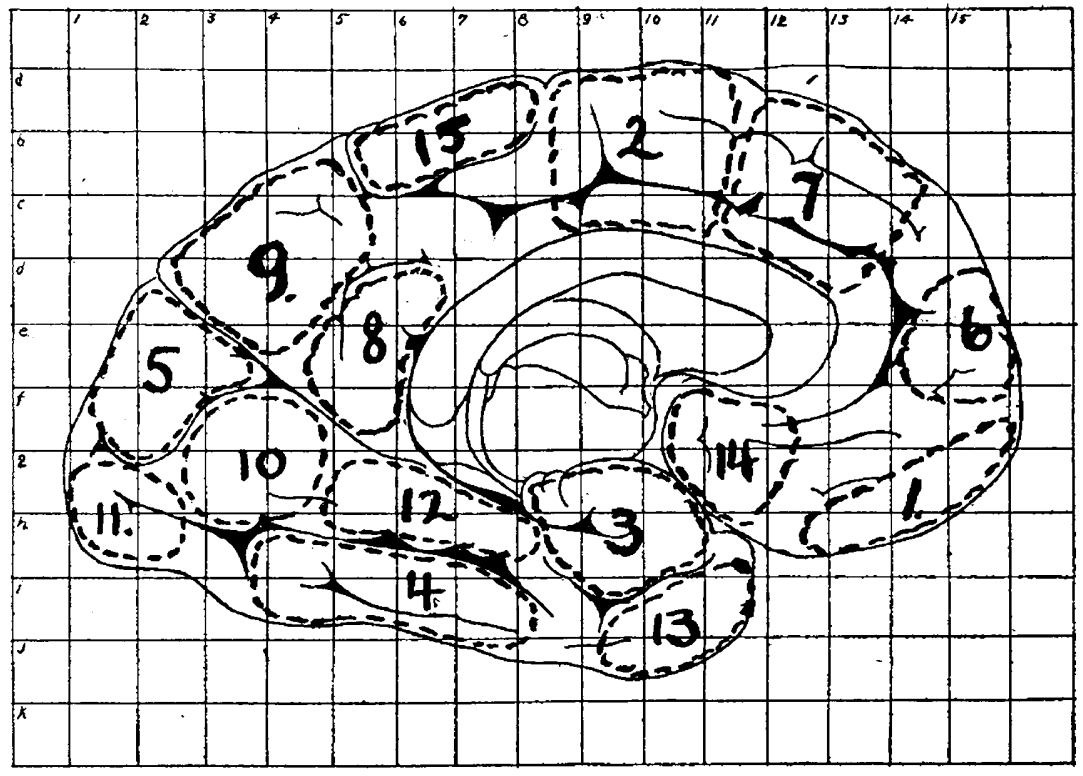

Fig. 2.-The mesial surface of the brain. 
TABLE 2.-Subcortical Structures Under III and IV, Table 1

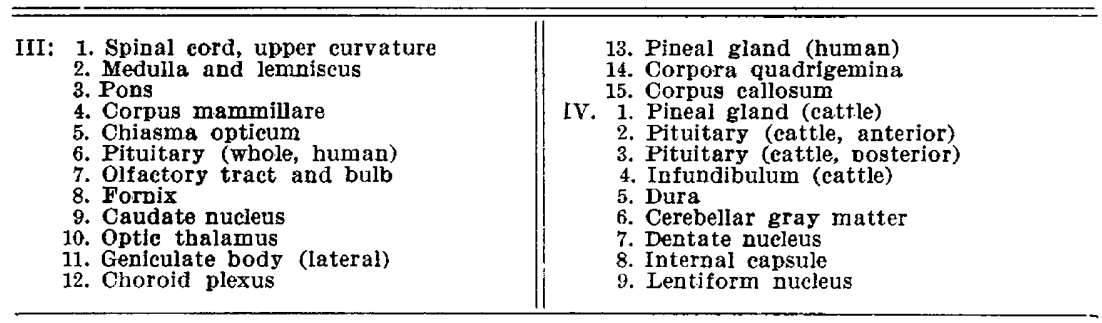

\section{APPARATUS AND TECHNIC}

Dialyzers.-They are made of collodion in the following way: A 10 per cent. solution of well dried celloidin shreds in equal parts of alcohol and ether is filled into tubes $3 / 4$ by 4 inches (best with far outstanding rims; so-called fat melting point tubes). The tube is now reversed and the collodion is allowed to flow directly back into the bottle for one minute. The tube is then reversed again and allowed to stand another minute upright, until a small drop collects on the bottom of the tube. The tube is reversed again, the ring of collodion permitted to reach about the half of the height of the tube and turned back into position. Through turning of the tube the solution is now distributed evenly on the lower part of the tube. After a short experience one is able to judge just how much of the solution to let accumulate on the bottom in order to obtain a good thin tube with enough strength in the bottom to prevent folding in.

The ether vapor is blown out from the inside of the tube, which next is filled with 80 per cent. alcohol. After allowing it to stand for about twenty minutes, the alchol is poured out and the tube, with the collodion film, immersed in cool running water. After all the alcohol is washed out, which takes place after about thirty minutes, the rim is loosened, and the dialyzer can now be easily pulled out. If the dialyzer made in this way has no air bubbles and is evenly shaped, it can be used without testing. It is advisable to reject without test all those which are either uneven or too white (they ought to be just slightly milky), or if they contain in the wall, and especially on the bottom, any air bubbles. There is too much danger of breaking the very much thinner wall of air bubbles and making the dialyzer leak and consequently losing the experiment. The dialyzers are preserved in 80 per cent. alcohol and washed for half an hour in running water before every test. They can be used over and over again, only care must be taken to remove all the serum after every use. For that end it is advisable to wash them first in physiologic sodium chlorid solution to prevent any globulins from precipitating, and afterward in running water for at least one hour. They ought to be in alcohol for at least six hours between tests in order to sterilize them.

Dialyzers made in this way keep very well for many months; are ready for use at short notice, and are uniform in action.

The way of making them was adapted, with some changes, from a similar method by Pregl. ${ }^{2}$

Dialyzing Flasks.-Instead of the expensive Erlenmeyers with wide neck, as recommended by Abderhalden, I am using ordinary wide-mouthed, tallform 3-ounce bottles. The advantage of these bottles is the low price, and what is more important, the possibility of utilizing a much taller column of water for dialysis, thus proportionally to the dialyzing surface increasing the rate of dialysis.

2. Pregl: Fermentforschung, 1914, 1, 1. 
Water.-Ordinary distilled water (neutral to litmus) is used.

Serum.-The blood is usually taken from any of the large veins in the arm with just enough constriction not to suppress the pulse entirely. A short needle, gage 16, was found most suitable because, while not making too big a hole in the vessel, it allows the collection of the necessary volume of blood quickly, thus preventing any annoyance to the patient. About 3 or 4 ounces of blood are collected into dry sterile bottles without the use of any anticoagulant. The blood is allowed to stand for an hour or so at room temperature, then the clot is torn to smaller pieces with sterile long hatpins and separated well from the glass, and the container left in the ice-box for several hours (over night). The serum is poured off into centrifuge tubes and centrifugated at medium speed for about ten minutes. The clear serum is pipetted off into a dry sterile graduated cylinder.

The serum should not show much hemoglobin, although that does not interfere markedly with the reaction, according to my experience. In the same way it is not essential to take blood outside of the digestive period, though it seems to give a clearer cut experiment to do so. Presence of bile in blood does not interfere with the reaction, beyond staining the dialyzers.

Substrates.-As material for my brain tissues, human brains from necropsies were used. Only brains from individuals perfectly normal, as far as the brain was concerned, were used and care was taken to use as fresh material as possible (not more than ten hours after death) to avoid postmortem changes in the tissues.

All the large vessels were removed and pieces cut out as marked in Table 2 and Figures 1 and 2. In slicing off pieces from the cortex, care was taken to remove only the gray matter, the parts from inside the fissures not being utilized at all. The pieces were cut into small particles of the size of a small pea and left stand for some time in labeled flasks filled with tap water, preferably in the ice-box. Every two hours the water was changed until all traces of blood disappeared. Now distilled water was used in the same way, and lastly distilled water with 0.5 per cent. acetic acid. After all the blood was removed from the tissue in this way, the pieces were coagulated by immersing for about two minutes in boiling water. The tissue was fished out, put back into the labeled flask and extracted cold two or three times with alcohol and as many times with ether in order to remove all fat and lipoids. When a drop of extracting fluid does not leave any fat on evaporation on a watch glass, the tissue is ready for freeing it from dialyzable substances giving the ninhydrin reaction. That is accomplished by immersing the particles in boiling water for not more than five minutes and repeating the procedure several times with fresh water. Care must be taken not to prolong the boiling too much, because then we run the danger of hydrolyzing or boiling off the specific protein. The boiling is repeated until 10 c.c. of the water in which the tissue was boiled ceases to give a reaction with 0.2 c.c. of a 1 per cent. ninhydrin solution on boiling for about three minutes; that is, until about 2 c.c. are left in the tube. The tissue is now transferred to sterile bottles, covered with boiling distilled water and about $1 / 2$ inch of toluene. If the bottles are properly corked and the pieces, when necessary, taken out under aseptic conditions, the tissues thus prepared keep almost indefinitely. Just before the test the substrate is boiled three times, for a short while each, in distilled water.

Test Tubes.-The test tubes should not alkalinize the water on boiling and therefore only the best quality should be used-Jena, Nonsol, or best of all, Pyrex. The test tubes and the flasks are always cleaned with chromic acid.

The Test.-Into each of the dialyzing bottles 10 c.c. of neutral distilled water is measured with a pipet. The washed dialyzer is taken into the left (scrubbed) hand, a piece of the chosen substrate inserted into it carefully with a long forceps and from 0.7 to 1 c.c. of the clear serum added. The charged dialyzer, after. being provided with a half inch layer of toluene over the 
serum, is put into the bottle, a few drops of toluene dropped on the water and all covered with a watch glass. As many dialyzers are charged as different substrates are to be used and every bottle is labeled to avoid confusion. Besides those tissue dialyzers, an odd number (three or five) of serum control tubes (without any substrates) are rigged up in the same way. All bottles are left for twenty hours in an incubator regulated at between 26 and $30 \mathrm{C}$. This low temperature was chosen, as it is sufficient for the digestion, while it is not high enough to spoil the dialyzers by promoting in any very marked degree the hydrolysis of celloidin, which, being an ester, is hydrolyzible.

After twenty hours' incubation the water from the outside of the dialyzer is transferred into alkali-free test tubes. The contents are boiled for a short time to drive off the toluene, 2 drops of a 1 per cent. solution of ninhydrin are added and the contents of the tube again boiled briskly until only a $1 / 2$ inch high layer is left in the test tube. If, in the case of bumping, or foaming, the liquid becomes turbid or precipitates, the test is rejected because of the possibility of the presence of protein due to a leaky dialyzer. If, on the other hand, the liquid remains clear but refuses to boil quietly, one or more short glass capillaries (sealed on the upper end) may be dropped into the tube.

The reading is made at least half an hour after the last tube is boiled, in which time the equilibrium is surely reached. The reading is made by comparison with the control tubes which sometimes are slightly colored. This may be due either to presence in the serum of digestive ferments, or aminoacids and peptones, if the blood was taken during digestion or was slightly hemolyzed. After a short experience this fact does not make any difficulties. The control tube is considered negative. Three plus was the designation given to the color which appears when 5 c.c. of a 0.1 per cent. solution of asparagin and 2 drops of the ninhydrin are added to a boiled down control tube, and the contents boiled as in all other tests. The intermediate colors are called 2 and 1 plus.

After the ninhydrin test is read the reactions are painted with colored inks -white, blue, violet, and red ( 3 plus) -on charts identical with Figures 1 and 2 , as well as a figure indicating the subcortical structures of Table 2, but printed on transparent paper, thus showing after overimposing in transmitting light all the reactions in various levels at a time, and allowing the determination of the localization of the lesion.

\section{RESULTS OF TESTS}

CAse 1.-W. V. Psychopathic Hospital, Chicago, Jan. 3, 1916. Brain tumor. Only seven different brain tissues were used as substrates. In this and the two following experiments, a mixture of all the parts of the cortex was used as substrate without differentiation. An exceedingly strong reaction with the pituitary tells the localization. Clinically, acromegaly.

Case 2.-Mr. P. Psychopathic Hospital, Chicago, Feb. 8, 1916. Brain tumor. Ten different substrates were used. The reaction with the anterior pituitary ( 3 plus) indicates the localization of the lesion, as would be expected, according to a clinical diagnosis of acromegaly.

CASE 3.-Mr. N. Cook County Hospital, Feb. 8, 1916. Clinical Diagnosis, brain tumor. Nine different substrates were used. The cerebellar gray matter and the pons gave strong positive reactions. A necropsy performed a week later by Dr. Bissel, pathologist of Cook County Hospital, revealed a tumor in the cerebellopontine angle.

CASE 4.-A. F. Presbyterian Hospital, Chicago. Dr. R. Wilder, June 8, 1916. Clinical diagnosis known to me; pineal gland disease (sexual precosity). Fourteen substrates were used. The double negative reaction with the pineal gland excludes that structure. The lesion is localized in the posterior lobe of the hypophysis $\left(\mathrm{III}_{\mathfrak{e}}\right.$ and $\mathrm{IV}_{\boldsymbol{s}}$ ) representing probably hypofunction, which would 
produce sexual symptoms similar to those due to pineal trouble. Clinical diagnosis afterward changed to posterior pituitary insufficiency.

CASE 5. - L. D. Cook County Hospital. Dr. R. Hamill, May 18, 1916. Clinical findings, motor aphasia and right body hemiparesis. Eighteen different substrates were used. The most pronounced reactions are in the optic thalamus, pons, frontal cortex and the Broca convolution.

CASE 5a.-Same case. June 22, 1916, shows practically the same reactions, with only slight differences in intensity of positiveness.

CASE 6.-J. F. Presbyterian Hospital, Chicago. Dr. D. Lewis, June 23, 1916. Brain tumor. Fourteen different substrates used. The most important reactions are in the pituitary ( $\mathrm{III}_{\sigma}-3$ plus) and especially its frontal lobe $\left(\mathrm{IV}_{\mathrm{s}}-3\right.$ plus) and the occipital vision center ( $\mathrm{II}_{5}-2$ plus). Localization: The lesion is in the anterior hypophysis; visual trouble. Clinically, acromegaly; blindness.

CASE 7.-G. J. Psychopathic Hospital, Chicago, April 16, 1917. Twentyone different tissues used as brain substrates. The reaction ( 3 plus) with the occipital vision center $\left(\mathrm{II}_{5}\right)$, and the anterior pituitary $\left(\mathrm{IV}_{2}-3\right.$ plus) suggest the localization of the lesion in the hypophysis. A subsequent roentgen-ray examination reveals a very small sella turcica, posterior clinoid processes much enlarged and possible pituitary hypoplasia. Clinically, visual delusions.

CASE 8.-Em. F. May 24, 1917. Twenty-one different substrates were used. Besides a 2 plus reaction in the pituitary (anterior), there is a prominent reaction with the visual center in the occipital lobe. The patient later gave a history of having suffered several years from double vision, but the relation of this to the reaction, if any, is not clear.

CAse 9.-Mr. T. Psychopathic Hospital, Chicago, Aug. 8, 1917. Clinical diagnosis, violent mania with visual hallucinations. Forty different substrates

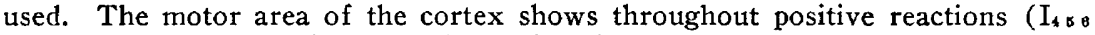
and $\mathrm{II}_{2}$ ), while the positive reactions with visual path represented by the cuneus ( $\mathrm{II}_{5}-2$ plus) the corpora quadrigemina ( $\mathrm{III}_{14}-2$ plus) and the lateral geniculate bodies ( $\mathrm{III}_{11}-3$ plus) correspond with the visual delusions.

CAse 10.-A. G. Dr. A. Heym, Nov. 9, 1917. Diagnosis, brain tumor. Thirtyfour different substrates were used. The motor area in the cortex shows very pronounced positive reactions $\left(\mathrm{I}_{4 \mathrm{~B}}\right)$. The pituitary gives strong reactions (2 plus- $\left.-\mathrm{II}_{6}, \mathrm{IV}_{2}\right)$. The lesion lies in the anterior lobe of the pituitary. Clinical diagnosis, acromegaly with occasional epileptiform attacks.

Case 11.-J. M. Psychopathic Hospital, Chicago, Nov. 26, 1917. Dr. G. W. Hall. Clinical diagnosis, tumor in the left frontal lobe. Thirty-four different substrates were used. The lesion was apparently cortical, because all noncortical tissues reacted negatively (III and IV) and lies clearly in the frontal lobe $\left(I_{12 \text { s }}\right.$ are 3 plus positive). Because of too small a number of different parts of the frontal cortex used for the test, no nearer localization could be attempted. The clinical findings, in short, were the following: Trauma three months previously; rapid mental deterioration for the previous month; disorientation; emotionalism; recognized objects but could not name them; general tremor; jerking of right arm at times. In the beginning of February the patient died after a second trephining (in the left frontal region) and on necropsy, performed by Dr. LeCount, was found a glioma of the left parietal lobe in the neighborhood of the base of the temporal lobe.

Case 12.-F. A. Presbyterian Hospital, Chicago, Dec. 6, 1917. Dr. Thor Rothstein. Diagnosis, brain tumor. Fifty-nine different substrates were used. All the positive reactions in the frontal, central, parietal and occipital lobes could be centered by the association tracts in the temporal lobe and especially in its basal part. Here, too, were the two most pronounced reactions ( $I_{0}$ and II.). Pathology in that region would very well explain the reaction with the corpora quadrigemina $\left(\mathrm{III}_{14}\right)$ and the cerebellar gray matter $\left(\mathrm{IV}_{8}\right)$. It seems 
that the lesion was located in the base of the temporal lobe in its white matter and that the pressure was directed mostly toward the adjoining parts of the cerebellum and possibly the corpora quadrigemina.

The clinical findings related to me after finishing the test were the following: Pupils large and equal; right does not respond to light or accommodation; left sluggishly; vision in right practically absent; distinct temporal hemianopsia in the left; no evidence of involvement of cranial nerves; rigid neck; passive motion in all directions causes pain, which is also the cause of difficulty in rising from recumbent position; vomiting; fainting spells; all reflexes normal; spinal pressure 265 . Clinical diagnosis, tumor of the hypophysis. Since the patient left the hospital without being operated on, neither the clinical nor the serologic diagnosis culd be confirmed.

Case 13.-S. S. Cook County Hospital, Jan. 28, 1918. Dr. G. W. Hall. Diagnosis, probably brain tumor. Fifty-nine different substrates were used. The lesion was evidently cortical, since all the most pronounced reactions appeared in the convex surface $\left(\mathrm{I}_{23_{\text {a }} 1320-3}\right.$ plus $)$. All of these areas could be centralized through the association fibers in $I_{3}$. Hence the localization of the lesion in the upper part of the third frontal convolution. Clinically, tumor in the right frontal lobe.

CASE 14.-Stan. G. Cook County Hospital, Jan. 28, 1918. Dr. Hamill. Aphasia and right body hemiplegia. Fifty-seven different substrates were used. The 3 plus reaction with the Broca convolution suggested aphasia. Three plus reactions with the lower anterior central convolution is accounted for, on one hand, by the strong reaction with the internal capsule ( 2 plus- $\left.\mathrm{IV}_{8}\right)$, on the other hand, by an apparent degeneration of fibers connecting the motor area with the base of the caput nuclei caudati ( 3 plus- $\mathrm{III}_{y}$ ) and running thence through the corpus subthalamicum and the lemniscus (III -1 plus).

Thus the lesion, of whatever nature it is, must lie in the lower portion of the anterior central gyrus and Broca's convolution, causing aphasia, right body hemiplegia, and probably involvement of the fifth, seventh, and twelfth cranial nerves which originate in said area.

CASE 15.--V. B. Cook County Hospital, Feb. 11, 1918. Dr. Hamill. Aphasia. Sixty-one different substrates were used. The reaction shows diffuse changes following pretty closely the areas supplied by the middle cerebral artery and especially its external striate branch, so that only a hemorrhage or thrombosis can be suggested without any centralized localization. Clinical diagnosis, thrombosis involving the Broca convolution.

Case 16.-T. K. Aug. 31, 1917. Cook County Hospital. The following clinical diagnosis was given to me subsequent to the completion of the test: Sudden blindness from secondary optic atrophy; probable cause, serous meningitis.

The test was performed with forty different substrates. The strongest reaction ( 3 plus) was with the cuneus and corpora quadrigemina, 2 plus with the optic chiasm. In short, the test was strongly positive with the visual fibers of the brain, and indicated that some pathologic process involved these fibers. This entirely corresponded with the clinical symptoms as already stated.

CASE 17.-C. D. Sept. 24, 1917. Cook County Hospital. Dr. G. W. Hall. Clinical diagnosis, possible brain abscess or cerebral thrombosis.

The reactions, which were made with thirty-six different substrates, were, when positive, located almost only in the cortex and apparently scattered. The frontal lobe $\left(I_{1}-I_{2}-I_{5}-\right.$ see Fig. 1) proved to be strongly positive, though the corpus callosum, corpus mammillare, and the medulla reacted slightly positively. The path of the association indicated that the posterior part of the second frontal convolution was the probable seat of the lesion (point between $\left.I_{2}-I_{4-s}\right)$.

The necropsy performed by Dr. John Nuzum, pathologist of the Cook County Hospital, revealed a solitary abscess in exactly this place. 
Case 18.-H. S. Oct. 3, 1917. Presbyterian Hospital, Chicago. Dr. P. Bassoe. Clinical diagnosis, brain tumor. The reaction was tested with forty different substrates and indicated in the first place that the lesion was not in the cortex. The most pronounced reactions were with the cerebellar gray matter $\left(I_{\theta}\right)$, with the dentate nucleus $\left(I_{7}\right)$, anterior pituitary and the corpus callosum. The final diagnosis from the test was: lesion in the cerebellum, which was found at the operation in the form of a large cyst of the upper frontal part of the cerebellum.

CAse 19.-M. O'B. Presbyterian Hospital, Chicago. Dr. James B. Herrick. Clinical diagnosis, brain tumor. Forty substrates were used. The strongest reactions were with the posterior pituitary, with the chiasm the lateral geniculate bodies $\left(\mathrm{III}_{11}\right)$, the cuneus $\left(\mathrm{II}_{6}\right)$, the olfactory bulb $\left(\mathrm{III}_{7}\right)$ and the uncus $\left(\mathrm{II}_{3}\right)$.

The strong positive reaction with the optic chiasm explains the 2 plus with the vision center in the occipital lobe; especially because the reaction with the lateral geniculate body was 3 plus, thus completing the number of positive reactions throughout the central visual path. The 2 plus reaction with the olfactory apparatus, due probably to pressure from the pituitary, suggests an olfactory disturbance, a fact which would explain the 2 plus reaction with the olfactory sensory area $\left(\mathrm{II}_{3}\right)$. This suggestion is still more upheld by the 2 plus reaction with the corpus mammillare $\left(\mathrm{III}_{4}\right)$, which, as known, holds some relation to the sense of smell. The reaction with the thalamus ( III $_{10}$ ) probably can be explained as involvement secondary to that of the corpus mammillare, possibly through the fasciculus thalamo-mammillare (bundle of Vica d'Azyr). The 2 plus reaction with the choroid plexus would indicate intracranial pressure. Thus the localization of the lesion in the posterior pituitary.

After delivery of the complete report the following clinical and laboratory details were given: Diagnosis of tumor in the hypophysis; roentgen-ray examination shows great destruction in the sella turcica. Ophthalmologic examination: Visual field of left side destroyed almost completely; in the right eye very much subnormal. Almost complete loss of smell on the left side as compared with the right side.

CASE 20.-Mrs. H. Oct. 18, 1917. Presbyterian Hospital, Chicago. Dr. P. Bassoe. Clinical diagnosis, brain tumor. Forty different substrates were used. The most pronounced reactions were in the fornix $\left(\mathrm{III}_{8}\right)$, the optic chiasm $\left(\mathrm{III}_{5}\right)$ and the anterior pituitary $\left(\mathrm{IV}_{2}\right) ; 2$ plus reactions in the optic thalamus (III ${ }_{10}$ ) and the choroid plexus ( $\mathrm{III}_{12}$ ), a weak reaction with the caudate nucleus (III)), the other part of the hypophysis $\left(\mathrm{IV}_{3}\right)$, the olfactory apparatus (III $)$ and the corpus callosum ( $\mathrm{III}_{15}$ ).

Since there was a general diagnosis of tumor, an attempt was made to localize it in one of the enumerated structures. A lesion in the pituitary or in the chiasm causing such strong reaction would undoubtedly cause visual disturbances and reflect on the geniculate bodies, corpora quadrigemina and the visual center in the sensory area of the occipital lobe; since these parts gave negative reactions the localization was to be in the fornix or the thalamus, and it was decided that it was most likely in the fornix, because a lesion in the thalamus probably would have brought about secondarily some cortical reactions which are missing.

After delivery of my report and localization I was informed that clinically there was no localized lesion, but an internal hydrocephalus of the third ventrical with pressure directed mostly toward its frontal wall. The reactions obtained in the test, as can be seen, are in no way contradictory to that diagnosis. Only the posterior wall of the third ventricle-the corpora quadrigemina and the pineal body-are represented by a negative reaction, while all its frontal, superior and lateral structures gave strong reactions that indicate their involvement possibly from pressure. Some time after this test the patient had a left temporal decompression performed, and since then developed signs indicating tumor in the right hemisphere. 
CASE 21.-M. K. Cook County Hospital, Oct. 23, 1917. Service of Dr. J. Grinker. Clinical diagnosis, tumor or abscess of the cerebellopontine angle. This clinical diagnosis was known to me when this test was made.

Thirty-eight different tissues were used. The five weak reactions in the cortex (Table 1) are in apparently no direct relation to each other and exclude the idea of a cortical lesion, but the strong reaction ( 3 plus) with corpus callosum ( $\left(\mathrm{II}_{15}\right)$ suggests that those reactions are bilateral. The basal ganglia, with the sole exception of the pineal gland, were negative. Thus the remaining positively reacting structure-the pons ( III $_{5}$ ) - must be the seat of the lesion, while the 3 plus with the choroid plexus $\left(\mathrm{III}_{12}\right.$ ) indicates probably increased intracranial pressure which led to the clinical diagnosis of brain tumor. The clinical localization in the cerebellopontine angle was based chiefly on the involvement of cranial nerves from III and XII. The necropsy performed on Nov. 10, 1917, by Dr. J. Nuzum, showed pressure on the pons by a large tumor from the nasopharynx.

CAse 22.-Mrs. G. Oct. 22, 1917. Michael Reese Hospital, Chicago. Dr. S. Kuh. Clinical diagnosis, brain tumor. Thirty-eight different substrates used. The whole aspect of the reaction points to two areas of possible lesion-the frontal lobe and the corpora quadrigemina. If we consider the lesion in the frontal convolution, then it would be expected that the corpora quadrigemina would be involved too, through the degeneration of corresponding association fibers, while if the primary lesion were to be in the corpora quadrigemina the reaction with the chiasm (IIIs) would naturally have to be stronger than found. In addition, the prevalence of strong reactions alone points to the localization of the lesion being in the frontal lobe; probably the posterior part of the second frontal convolution.

After receiving this serologic localization, Dr. Kuh disclosed his clinical findings, which led him to the localization of the tumor in the second frontal convolution.

CASE 23.-H. G. Oct. 23, 1917. Cook County Hospital. Dr. G. W. Hall. Clinical diagnosis, brain tumor. The number of positive reactions in the subcortical regions suggested the making of an attempt to localize the tumor outside of the hemispheres. On account of that, the fibers running from all cortex areas showing positive reactions were traced. The first thing which attracts the attention is the involvement of the cortical origin of the central path of cranial nerves III and XII (with the exception of the accessorius), the cortical origin of nerves V and XII is located in the lower two-thirds of the anterior central gyrus and is here represented by positive reactions ( $I_{5}$ and $\left.I_{6}\right)$, while the oculomotor and trochlear originate in the superior portion of the second frontal convolution which gave a 3 plus reaction $\left(I_{2}\right)$. The nuclei of all these nerves give a positive reaction ( III $_{2}$ ) and it is to be remembered that the nuclei of the third and fourth cranial nerves lie nearest to the walls of the fourth ventricle and that they derive from a cortex area giving a 3 plus reaction; the corpora quadrigemina are 2 plus positive, and the centrifugal fibers from the temporal $\left(I_{v}\right)$ and occipital lobe $\left(I_{10}\right)$ run through the posterior commissure, the nearest structure to the corpora quadrigemina; the choroid plexus $\left(\mathrm{III}_{12}\right)$, the cerebellar gray matter $\left(\mathrm{IV}_{6}\right)$ and the dentate nucleus $\left(\mathrm{IV}_{7}\right)$ all gave a very distinct positive reaction.

On the basis of this analysis the lesion was localized in the fourth ventricle, with the involvement of the dentate nucleus and the gray matter of the cerebellum, of the aqueduct of Sylvius and of the corpora quadrigemina as far as the pineal gland. A possibility of a second focus in the hypophysis was pointed out $\left(\mathrm{IV}_{23}\right)$.

The clinical diagnosis was (as disclosed after the tests were reported) tumor in the region of the lower temporal lobe of the right side, with pressure involving the corpora quadrigemina. 
The necropsy performed Oct. 26, 1917, by Dr. J. Nuzum revealed a large soft glioma beginning in the dentate nucleus, and extending over the corpora quadrigemina. The hypophysis was undermined by a cyst.

CASE 24.-Mr. X. Nov. 9, 1917. Private patient of Dr. B. G. Hassin. Diagnosis, well localized brain lesion. The result of the test (Table 1) made with thirty-six different substrates did not admit of any other diagnosis than that of a lesion in the upper motor area $\left(\mathrm{I}_{124}\right)$. The clinical diagnosis, received as usual after the delivery of the serologic report, was: Jacksonian epilepsy confined to the left leg only, due to an old skull trauma.

CASE 25.-Mr. XX. Nov. 16, 1917. Private patient of Dr. A. Heym. Diagnosis, epilepsy possibly due to a localized lesion. Thirty-seven different substrates used. The test shows besides rather weak reactions in the parietal and temporal lobes a very strong reaction ( 3 plus) over the whole extent of the anterior central convolution $\left(\mathrm{I}_{4-8}\right)$ and the paracentral gyrus $\left(\mathrm{II}_{2}\right)$.

The strong positive reactions with the caudate nucleus and corpus mammillare ( 3 plus- $\mathrm{III}_{9}, \mathrm{IV}_{4}$ ) are explained by the fact that the fasciculus subcallosus, which originates in the anterior central convolution, is interrupted in the caudate nucleus, while the central cortical path of the tegmentum, which originates in the same cortical area, after crossing the caudate and lentiform nuclei passes into the neighborhood of the corpus mammillare.

On the basis of the serologic findings the lesion was localized in the upper part of the central convolution. An operation performed ten days later revealed a deep tumor of the size of a hazelnut, which could be easily palpated in this location.

In this brief report the discussion of the individual cases has been purposely omitted and only the most important and striking features have been noted. On the basis of the findings in the limited series, the serologic diagnosis is supported in four cases by the post mortem findings, in two cases by an operation, and in fifteen cases the serologic findings are in complete agreement with definite clinical evidence. Two cases differ from the clinical diagnosis, but the diagnosis has not been controlled by necropsy or operation. In one case (Case 11) the necropsy findings proved that the serologic diagnosis was erroneous. 